\title{
Effect of Exotic Species on a System of Native Prey-Predator Populations : A Model
}

\author{
O. P. Misra ${ }^{1}$, Pramod Kushwah ${ }^{2, *}$, Chhatrapal Singh Sikarwar ${ }^{1}$ \\ ${ }^{1}$ School of Mathematics and Allied Science, Jiwaji University, Gwalior (M.P.), 474011, India \\ ${ }^{2}$ Govt. M.J.S.P.G. College, Bhind (M.P.), 477001, India
}

\begin{abstract}
In this paper, a mathematical model is proposed to study the effect of exotic predator population on a system of native prey-predator population. The model includes three state variables viz., density of native prey, density of native predator and density of exotic predator. The stability analysis of all the feasible equilibria are carried out and also the possibility of Hopf- bifurcation of the interior equilibrium point is investigated for the parameter $a_{2}$; the predation rate of exotic predator. By vary ing the parameter $a_{2}$, a change in stability behaviour of the interior equilibrium is also observed. The stability and direction of bifurcating periodic solution is discussed. Finally the analytical results are supported by numerical simulation.
\end{abstract}

Keywo rds Biological Invasion, Equilibria, Stability, Hopf-Bifurcation, Predation Rate

\section{Introduction}

The prey predator relationship still continues to be one of the main themes in mathematical ecology due to its complex dynamic behavior. Many prey predator models have been studied considering different types of functional responses $[1,2,3]$. It is seen that the invasion or introduction of exotic species in general disrupt the trophic dynamics of native interacting prey-predator species systems.

Introduced predators usually have a dramatic effect on native prey, usually the cause of native species ext inction[4, $5,6]$. The harm caused by the introduced predators is broadly known and control programs are largely identified as the best way to restore ecosystems[7]. Many authors have studied effect of exotic predator on native prey species $[8,9,10]$.

Dynamical consequences of predator interference in a tri-trophic model food chain is investigated by R K Naji et al[11]. Three-species food-chain model with Beddington DeAngelis type functional response has been studied by Wang and Zhao[12].

Meng Fan et al.[9] investigated the dynamical interaction among prey (bird), mesopredator (rat), and superpredator (cat) and developed a prey-mesopredator-superpredator (i.e. bird-rat-cat) BRC model, where the predator's functional response is derived based on classical Holling's time budget arguments. They have explored possible control strategies to save or restore the bird by controlling or eliminating the rat or the cat when the bird is endangered. They do not show

* Corresponding author: pramod.kushwah@rediffmail.com (Pramod Kushwah) Published online at http://journal.sapub.org/ajcam Copyright (C) 2012 Scientific \& Academic Publishing. All Rights Reserved under what conditions all the three species (BRC) will coexist and global stability analysis of interior equilibrium point is also not carried out.

In view of the above, the main purpose of this paper is to construct a general model to study the effect of exotic predator on a system consisting of a native prey population and a native predator population by considering Holling type two functional response with exotic predator interference for native predator population and Beddington type functional response for exotic predator population.

\section{Basic Assumptions and Mathematical Model}

Let $S(t)$ denotes the density of native prey population, $R(t)$ denotes the density of native predator population and $C(t)$ denotes the density of exotic predator population. We assume that $r$ and $k$ are growth rate and carrying capacity of native prey population respectively. $d_{1}$ and $d_{2}$ are death rate of native predator population and exotic predator population respectively. $b$ is the interference due to exotic predator population. $a_{1}$ is the predation rate of native prey population by native predator population. $b_{1}$ is the growth rate of native predator population due to predation of native prey population. $a_{2}$ is the predation rate of native predator population by exotic predator population. $b_{2}$ is the growth rate of exotic predator population due to predation of native predator population. $h$ is the half saturation constant of native prey population. $w$ is the wasting time to searching native predator by exotic predator. $h_{1}$ is the handling time to handle native predator by exotic predator. In view of above, the resultant system dynamics is governed by the following system of differential equations: 
Model 1 (With exotic species)

$$
\begin{gathered}
\frac{d S}{d t}=r S\left(1-\frac{S}{k}\right)-\left(\frac{a_{1} S}{h+S}\right)\left(\frac{R}{1+b C}\right) \\
\frac{d R}{d t}=\left(\frac{b_{1} S}{h+S}\right)\left(\frac{R}{1+b C}\right)-\frac{a_{2} R C}{1+\alpha R+\beta C}-d_{1} R \\
\frac{d C}{d t}=\frac{b_{2} R C}{1+\alpha R+\beta C}-d_{2} C
\end{gathered}
$$

With initial conditions $S(0) \geq 0, R(0) \geq 0$ and $C(0) \geq 0$.

where $b, r, k, h, \alpha=a_{2} h_{1}, \beta=a_{2} w, a_{1}, a_{2}, b_{1}, b_{2}, d_{1}$ and $d_{2}$ are positive constants.

In the absence of exotic predator species the above system (1) - (3) is governed by the following system of differential equations:

Model 2 ( Without exotic species)

$$
\begin{gathered}
\frac{d S}{d t}=r S\left(1-\frac{S}{k}\right)-\left(\frac{a_{1} S R}{h+S}\right) \\
\frac{d R}{d t}=\left(\frac{b_{1} S R}{h+S}\right)-d_{1} R
\end{gathered}
$$

With initial conditions $S(0) \geq 0$ and $R(0) \geq 0$

\section{Equillibria of the System}

In this section, we analy ze the system of equation (4) - (5) under the initial conditions. We find all the possible equillibria of the system of equation (4) - (5). The system has three feasible equillibria, namely

(i) Trivial equilibrium point $E_{T} \equiv(0,0)$

(ii) Axial equilibrium point $E_{A} \equiv(k, 0)$

(iii) Positive interior equilibrium point $E_{P} \equiv\left(S^{\#}, R^{\#}\right)$ where

$$
S^{\#}=\frac{d_{1} h}{b_{1}-d_{1}}, R^{\#}=\frac{r}{a_{1}}\left(h+S^{\#}\right)\left(1-\frac{S^{\#}}{k}\right)
$$

The positive equilibrium point $E_{P}$ exist if $b_{1}>d_{1}$.

We now analyze the system of equation (1) - (3) under the initial conditions. The system has four feasible equilibria, namely

(i)Trivial equilibrium point $E_{T_{e}} \equiv(0,0,0)$

(ii)A xial equilibriu m point $E_{A_{e}} \equiv(k, 0,0)$

(iii)Boundary equilibrium point $E_{B_{e}} \equiv\left(S^{\#}, R^{\#}, 0\right)$ where

$$
S^{\#}=\frac{d_{1} h}{b_{1}-d_{1}}, R^{\#}=\frac{r}{a_{1}}\left(h+S^{\#}\right)\left(1-\frac{S^{\#}}{k}\right)
$$

The boundary equilibrium point $E_{B_{e}}$ exist if $b_{1}>d_{1}$. (iv)Positive interior equilibrium point $E_{P_{e}} \equiv\left(S^{*}, R^{*}, C^{*}\right)$ where

$$
\begin{gathered}
S^{*}=\frac{S_{1}}{S_{2}-S_{3}} \\
R^{*}=\frac{r}{a_{1}}\left(1+b C^{*}\right)\left(h+S^{*}\right)\left(1-\frac{S^{*}}{k}\right) \\
C^{*}=\frac{\left(b_{2}-\alpha d_{2}\right) R^{*}-d_{2}}{d_{2} \beta} \\
S_{1}=h\left(1+b C^{*}\right)\left\{a_{2} C^{*}+d_{1}\left(1+\alpha R^{*}+\beta C^{*}\right)\right\}, \\
S_{2}=b_{1}\left(1+\alpha R^{*}+\beta C^{*}\right), \\
S_{3}=\left(1+b C^{*}\right)\left\{a_{2} C^{*}+d_{1}\left(1+\alpha R^{*}+\beta C^{*}\right)\right\},
\end{gathered}
$$

The Positive interior equilibrium point $E_{P_{e}}$ exist if $S_{2}>S_{3}$ and $b_{2} R^{*}>d_{2}\left(1+\alpha R^{*}\right)$.

Now, we will study the existence of positive interior equilibrium point $E_{P_{e}}$
Put value of $R^{*}$ from equation (7) in equation (6) we get,

$$
\begin{aligned}
& \qquad S^{*}=\frac{h H}{b_{1} b_{2}\left(1+\beta C^{*}\right)-H} \\
& \text { where } H=H_{1} C^{*^{2}}+H_{2} C^{*}+H_{3} \\
& H_{1}=b\left(a_{2} b_{2}-\alpha a_{2} d_{2}+\beta b_{2} d_{1}\right) \\
& \qquad H_{2}=a_{2} b_{2}-\alpha a_{2} d_{2}+\beta b_{2} d_{1}+b b_{2} d_{1}, H_{3}=b_{2} d_{1}
\end{aligned}
$$

Putting value of $R^{*}$ from equation (7) and putting value of $S^{*}$ from equation (9) in equation (8) we get

$$
B_{1} C^{*^{4}}+B_{2} C^{*^{3}}+B_{3} C^{*^{2}}+B_{4} C^{*}+B_{5}=0
$$

where $B_{1}=k a_{1} d_{2} H_{1}^{2}$;

$$
\begin{gathered}
B_{2}=2 H_{1}\left(H_{2}-\beta b_{1} b_{2}\right)+b h r b_{1} b_{2} H_{1}\left(b_{2}-\alpha d_{2}\right)(k+h) ; \\
B_{3}=2 b_{2} H_{1}\left(b_{1}-d_{1}\right)\left(H_{2}-\beta b_{1} b_{2}\right)^{2}+h r b_{1} b_{2}\left(b_{2}-\right. \\
\alpha d 2 k+h H 1+b H 2-b k \beta b 1 b 2 ; \\
B_{4}=2 b_{2}\left(b_{1}-d_{1}\right)\left(\beta b_{1} b_{2}-H_{2}\right)-h r b_{1} b_{2}\left(b_{2}-\right. \\
\alpha d 2 k b 1 b 2 b+\beta-k+h b b 2 d 1+H 2 ; \\
B_{5}=b_{2}^{2}\left(b_{1}-d_{1}\right)^{2}-r b_{1} b_{2}^{2} h_{1}\left(b_{2}-\alpha d_{2}\right)\left(k b_{1}-k d_{1}-\right. \\
d 1 h 1 .
\end{gathered}
$$

Therefore unique positive $\operatorname{root} C^{*}>0$ if $B_{2}>0, B_{3}>$ $0, B_{4}>0$ and $B_{5}<0$

\section{Dynamic Behaviour of the System}

The following results may be noted regarding the local stability of the equilibria of the model 2 given by (4)-(5).

(1). Equilibrium point $E_{T}$ of the model is unstable in the absence of exotic species.

(2). Equilibrium point $E_{A}$ of the model is stable when there is no exotic species present in the system if $\frac{b_{1} k}{h+k}<d_{1}$.

(3). Interior equilibrium point $E_{P}$ of the model is locally asymptotically stable when there is no exotic species present in the system if $\frac{d_{1}(h+k)}{k-h}>b_{1}$ is satisfied.

The following results may be noted regarding the local stability of the equilibria of the model 1 given by (1)-(4).

(1). Equilibrium point $E_{T_{e}}$ of the model is unstable in the presence of exotic species.

(2). Equilibrium point $E_{A_{e}}$ of the model is stable when exotic species is present in the system if $\frac{b_{1} k}{h+k}<d_{1}$.

(3). Equilibrium point $E_{B_{e}}$ of the model is stable when exotic species is present in the system if $\frac{b_{2} R^{\#}}{\left(1+\alpha R^{\#)}\right.}<d_{2}$ and $\frac{d_{1}(h+k)}{k-h}>b_{1}$ are satisfied.

(4). For the sake of simplicity, the equilibrium points $\left(S^{*}, R^{*}, C^{*}\right)$ of the system (1)-(3) is shifted to new points $\left(n_{1}, n_{2}, n_{3}\right)$ through transformations $n_{1}=S-S^{*}, n_{2}=$ $R-R^{*}, n_{3}=C-C^{*}$

In term of the new variables, the dyna mical system(1)-(3) can be written as in matrix form as

$$
\dot{X}=A X+B
$$

where dot cover $X$ denotes the derivative with respect to time. Here $A X$ is the linear part of the system and $B$ represents the nonlinear part. Moreover,

$$
X=\left[\begin{array}{l}
n_{1} \\
n_{2} \\
n_{3}
\end{array}\right], A=\left[\begin{array}{ccc}
a_{11} & a_{12} & a_{13} \\
a_{21} & a_{22} & a_{23} \\
0 & a_{32} & a_{33}
\end{array}\right],
$$




$$
B=\left[\begin{array}{c}
B_{11}+B_{12}+B_{13} \\
B_{21}+B_{22}+B_{23} \\
B_{31}+B_{32}
\end{array}\right\rceil
$$

where

$B_{11}=l_{1} n_{1}^{2}+l_{2} n_{1} n_{3}+l_{3} n_{1} n_{2}+l_{4} n_{3}^{2}+l_{5} n_{2} n_{3}$,

$B_{12}=l_{6} n_{1} n_{3}^{2}+l_{7} n_{1}^{2} n_{3}+l_{8} n_{1}^{3}+l_{9} n_{1} n_{2} n_{3}+l_{10} n_{1}^{2} n_{2}$,

$B_{13}=l_{11} n_{3}^{3}+l_{12} n_{2} n_{3}^{2}$,

$B_{21}=l_{13} n_{1} n_{2}+l_{14} n_{2}^{2}+l_{15} n_{2} n_{3}+l_{16} n_{3}^{2}+l_{17} n_{1} n_{3}$,

$B_{22}=l_{18} n_{1}^{2}+l_{19} n_{2} n_{3}^{2}+l_{20} n_{1} n_{2} n_{3}+l_{21} n_{1}^{2} n_{2}+l_{22} n_{2}^{3}$,

$B_{23}=l_{23} n_{2}^{2} n_{3}+l_{24} n_{3}^{3}+l_{25} n_{1} n_{3}^{2}+l_{26} n_{1}^{2} n_{3}+l_{27} n_{1}^{3}$,

$B_{31}=l_{28} n_{2}^{2}+l_{29} n_{2} n_{3}+l_{30} n_{3}^{2}+l_{31} n_{2}^{3}+l_{32} n_{3}^{3}$,

$B_{32}=l_{33} n_{2}^{2} n_{3}+l_{34} n_{2} n_{3}^{2}, a_{11}=\left(\frac{-r}{k}+a_{1} p_{2}^{2} p_{3} R^{*}\right) S^{*}$,

$a_{12}=-a_{1} p_{2} p_{3} S^{*}, a_{13}=a_{1} b_{1} p_{2} p_{3}^{2} S^{*} R^{*}$,

$a_{21}=h b_{1} p_{2}^{2} p_{3} R^{*}, a_{22}=a_{2} \alpha p_{1}^{2} R^{*} C^{*}$,

$a_{23}=-\left(b b_{1} p_{2} p_{3}^{2} S^{*} R^{*}+a_{2} p_{1}^{2} R^{*}\left(1+\alpha R^{*}\right)\right)$,

$a_{32}=b_{2} p_{1}^{2} C^{*}\left(1+\beta C^{*}\right), a_{33}=-b_{2} \beta p_{1}^{2} R^{*} C^{*}$,

$p_{1}=\left(1+\alpha R^{*}+\beta C^{*}\right)^{-1}, p_{2}=\left(h+S^{*}\right)^{-1}$,

$p_{3}=\left(1+b C^{*}\right)^{-1}$,

\begin{tabular}{|c|c|}
\hline$l_{1}=\frac{-r}{k}+a_{1} h p_{2}^{3} p_{3} R^{*}$ & $l_{2}=a_{1} b h p_{2}^{2} p_{3}^{2} R^{*}$ \\
\hline$l_{3}=-a_{1} h p_{2}^{2} p_{3}$ & $l_{4}=-a_{1} b^{2} p_{2} p_{3}^{3} R^{*} S^{*}$ \\
\hline$l_{5}=a_{1} b p_{2} p_{3}^{2} S^{*}$ & $l_{6}=-a_{1} b^{2} h p_{2}^{2} p_{3}^{3} R^{*}$ \\
\hline$l_{7}=-a_{1} b h p_{2}^{3} p_{3}^{2} R^{*}$ & $l_{8}=-a_{1} h p_{2}^{4} p_{3} R^{*}$ \\
\hline$l_{9}=a_{1} b h p_{2}^{2} p_{3}^{2}$ & $l_{10}=a_{1} h p_{2}^{3} p_{3}$ \\
\hline$l_{11}=a_{1} b^{3} p_{2} p_{3}^{4} R^{*} S^{*}$ & $l_{12}=-a_{1} b^{2} p_{2} p_{3}^{3} S^{*}$ \\
\hline$l_{13}=b_{1} h p_{2}^{2} p_{3}$ & $\begin{array}{l}l_{14} \\
=a_{2} \alpha p_{1}^{3} C^{*}\left(1+\beta C^{*}\right)\end{array}$ \\
\hline $\begin{array}{l}l_{15} \\
=a_{2} \alpha p_{1}^{2} R^{*}\left(1-2 \beta p_{1} C^{*}\right) \\
-a_{2} p_{1}^{2}\left(1+\alpha R^{*}\right) \\
-b b_{1} p_{2} p_{3}^{2} S^{*}\end{array}$ & $\begin{array}{l}l_{16} \\
=R^{*}\left(b_{1} b^{2} p_{2} p_{3}^{3} S^{*}\right. \\
\left.+a_{2} \beta p_{1}^{3}\left(1+\alpha R^{*}\right)\right)\end{array}$ \\
\hline$l_{17}=-b h b_{1} p_{2}^{2} p_{3}^{2} R^{*}$ & $l_{18}=-h b_{1} p_{2}^{3} p_{3} R^{*}$ \\
\hline $\begin{array}{l}l_{19} \\
=a_{2} \beta p_{1}^{4} R^{*}\left(1+\beta C^{*}\right. \\
\left.-\alpha^{2} R^{*^{2}}+2 \alpha \beta R^{*} C^{*}\right) \\
+b_{1} b^{2} p_{2} p_{3}^{3} S^{*}\end{array}$ & $\begin{array}{l}l_{20}=-b h b_{1} p_{2}^{2} p_{3}^{2} \\
l_{21}=-h b_{1} p_{2}^{3} p_{3}\end{array}$ \\
\hline$\stackrel{l_{22}}{=}-a_{2} \alpha^{2} p_{1}^{4} C^{*}\left(1+\beta C^{*}\right)$ & $\begin{array}{l}l_{23} \\
=\alpha a_{2} p_{1}^{4}\left(1+\alpha R^{*}\right. \\
\left.-\beta^{2} C^{*^{2}}+2 \alpha \beta R^{*} C^{*}\right)\end{array}$ \\
\hline $\begin{array}{l}l_{24} \\
=-R^{*}\left(a_{2} \beta^{2} p_{1}^{4}\left(1+\alpha R^{*}\right)\right. \\
\left.+b_{1} b^{3} p_{2} p_{3}^{4} S^{*}\right)\end{array}$ & $\begin{array}{l}l_{25}=b^{2} h b_{1} p_{2}^{2} p_{3}^{3} R^{*} \\
l_{26}=b h b_{1} p_{2}^{3} p_{3}^{2} R^{*}\end{array}$ \\
\hline$l_{27}=h b_{1} p_{2}^{4} p_{3} R^{*}$ & $\begin{array}{l}l_{28} \\
=-\alpha b_{2} p_{1}^{3} C^{*}\left(1+\beta C^{*}\right)\end{array}$ \\
\hline $\begin{array}{l}l_{29} \\
=b_{2} p_{1}^{3}\left(1+\alpha R^{*}+\beta C^{*}\right. \\
\left.+2 \alpha \beta R^{*} C^{*}\right)\end{array}$ & $\begin{array}{l}l_{30} \\
=-b_{2} \beta p_{1}^{3} R^{*}\left(1+\alpha R^{*}\right)\end{array}$ \\
\hline$\stackrel{l_{31}}{=} b_{2} \alpha^{2} p_{1}^{4} C^{*}\left(1+\beta C^{*}\right)$ & $\begin{array}{l}l_{32} \\
=b_{2} \beta^{2} p_{1}^{4} R^{*}\left(1+\alpha R^{*}\right)\end{array}$ \\
\hline $\begin{array}{l}l_{33} \\
=\alpha b_{2} p_{1}^{4}\left(\beta^{2} C^{*^{2}}-1\right. \\
\left.-\alpha R^{*}-2 \alpha \beta R^{*} C^{*}\right)\end{array}$ & $\begin{array}{l}l_{34} \\
=b_{2} \beta p_{1}^{4}\left(\alpha^{2} R^{*^{2}}-1\right. \\
\left.-\beta C^{*}-2 \alpha \beta R^{*} C^{*}\right)\end{array}$ \\
\hline
\end{tabular}

The eigen values of the matrix $A$ help to understand the stability of the system. The characteristic equation for the variational matrix $A$ is given by

$$
\lambda^{3}+A_{1} \lambda^{2}+A_{2} \lambda+A_{3}=0
$$

where

$$
\begin{gathered}
A_{1}=-\left(a_{11}+a_{22}+a_{33}\right), \\
A_{2}=a_{11}\left(a_{22}+a_{33}\right)+a_{22} a_{33}-a_{23} a_{32}-a_{12} a_{21}, \\
A_{3}=a_{11}\left(a_{23} a_{32}-a_{22} a_{33}\right)+a_{12} a_{21} a_{33}-a_{13} a_{32} a_{21},
\end{gathered}
$$

Using the Routh-Hurwitz criteria, we derive that the equilibrium point $E_{P_{e}}$ is locally asymptotically stable, if $A_{1}>0, A_{2}>0, A_{3}>0$ and $A_{1} A_{2}-A_{3}>0$ are being satis fied.

Now, we are in a position to make an attempt to find out the condition under which the system undergoes Hopf-bifurcation[13]. For this purpose, we choose the parameter ' $a_{2}$ ' as bifurcation parameter as it plays a crucial role to describe predation rate of native predator population by exotic predator population. We shall now apply the Liu's criteria[14] to obtain the conditions for small amplitude periodic solution arising from Hopf-bifurcation. As the equilibrium population densities are function of ' $a_{2}$ ', the coeffic ient of the characteristic equation (13) is a function of parameter ' $a_{2}$ ' and hence we can use the notation $A_{i}=$ $A_{i}\left(a_{2}\right)$ for $i=1,2,3$. Now noting that the quantities $A_{i}$ 's are smooth function of parameter ' $a_{2}$ ', we first state for our case, the definition of a simple Hopf-bifurcation.

If a crucial value $a_{2}^{*}$ of parameter ' $a_{2}$ ' is found such that (i) a simple pair of complex conjugate eigenvalues of characteristic equation (13) exist, say, $\lambda_{1}\left(a_{2}\right)=x\left(a_{2}\right)+$ $i y\left(a_{2}\right), \lambda_{2}\left(a_{2}\right)=x\left(a_{2}\right)-i y\left(a_{2}\right)=\overline{\lambda_{1}\left(a_{2}\right)}$ such that they becomes purely imaginary at $a_{2}=a_{2}^{*}$, i.e. $\lambda_{1}\left(a_{2}^{*}\right)=$ $i y^{*}, \lambda_{2}\left(a_{2}^{*}\right)=-i y^{*}$ with $y\left(a_{2}^{*}\right)=y^{*}>0$, whereas the other eigen value remain real and negative; and the transversality condition (ii) $\left(\left(d \operatorname{Re} \lambda_{i}\left(a_{2}\right)\right)\right) /\left.d a_{2}\right|_{a_{2}=a_{2}^{*}}=$ $\left.\left(\left(d x\left(a_{2}\right)\right) / d a_{2}\right)\right|_{a_{2}=a_{2}^{*}} \neq 0$ is satisfied, then at $a_{2}=a_{2}^{*}$, we have a simple Hopf-bifurcation. Without knowing eigen value[14] proved that (referring the result to the current case): if $A_{1}\left(a_{2}\right), A_{3}\left(a_{2}\right), \Delta\left(a_{2}\right)=A_{1}\left(a_{2}\right) A_{2}\left(a_{2}\right)-A_{3}\left(a_{2}\right)$ are smooth function of the parameter ' $a_{2}$ ' in an open interval containing $a_{2}^{*} \in R_{+}$such that the following condition hold.

$$
\begin{gathered}
\left(i_{*}\right) A_{1}\left(a_{2}^{*}\right)>0, A_{3}\left(a_{2}^{*}\right)>0, \Delta\left(a_{2}^{*}\right)=0 \\
\left.\left(i i_{*}\right) \frac{d \Delta\left(a_{2}\right)}{d a_{2}}\right|_{a_{2}=a_{2}^{*}} \neq 0
\end{gathered}
$$

Then $\left(i_{*}\right)$ and $\left(i i_{*}\right)$ are equivalent to conditions $(i)$ and (ii) for the occurrence of a simple Hopf-bifurcation at $a_{2}=a_{2}^{*}$. Hence we can propose the following theorem:

Theorem 4.1 If a critical value $a_{2}^{*}$ of parameter ' $a_{2}$ ' is found such that $A_{1}\left(a_{2}^{*}\right)>0, A_{1}\left(a_{2}^{*}\right)>0, \Delta\left(a_{2}^{*}\right)=0$ and further $\Delta^{\prime} \neq 0$ (where prime denotes differentiation with respect to $a_{2}$ ) then system (1)-(3) undergoes Hopf-bifurcation around $E_{P_{e}}\left(S^{*}, R^{*}, C^{*}\right)$.

Next, we seek a transformation matrix $P$ which reduces the matrix $A$ to the form

$$
P^{-1} A P=\left[\begin{array}{ccc}
0 & -v & 0 \\
v & 0 & 0 \\
0 & 0 & -A_{1}
\end{array}\right]
$$


Where the non-singular matrix $P$ is given as

$$
P=\left[\begin{array}{ccc}
P_{11} & P_{12} & P_{13} \\
P_{21} & P_{22} & P_{23} \\
P_{31} & 0 & P_{33}
\end{array}\right]
$$

where, $P_{11}=a_{22} a_{33}-a_{23} a_{32}-v^{2}, P_{12}=v\left(a_{22}+a_{33}\right)$,

$P_{13}=\left(a_{22}+A_{1}\right)\left(a_{33}+A_{1}\right)-a_{23} a_{32}, P_{21}=-a_{21} a_{33}$,

$P_{22}=-v a_{21}, P_{23}=-a_{21}\left(a_{33}+A_{1}\right), P_{31}=a_{21} a_{32}$,

$P_{33}=a_{21} a_{32}$

To achieve the normal form of (10), we make another change of variable, that is,

$$
X=P Y \text {, where } Y=\left[\begin{array}{l}
y_{1} \\
y_{2} \\
y_{3}
\end{array}\right]
$$

Through some algebraic manipulation, (10) takes the form

$$
\dot{Y}=\Omega Y+F
$$

where $\Omega=P^{-1} A P$ and

$$
\begin{gathered}
F=P^{-1} f=\left[\begin{array}{l}
F^{1}\left(y_{1}, y_{2}, y_{3}\right) \\
F^{2}\left(y_{1}, y_{2}, y_{3}\right) \\
F^{3}\left(y_{1}, y_{2}, y_{3}\right)
\end{array}\right], f \text { is given by } \\
f=\left[\begin{array}{l}
f^{1}\left(y_{1}, y_{2}, y_{3}\right) \\
f^{2}\left(y_{1}, y_{2}, y_{3}\right) \\
f^{3}\left(y_{1}, y_{2}, y_{3}\right)
\end{array}\right]
\end{gathered}
$$

where,

$$
\begin{aligned}
f^{1}\left(y_{1}, y_{2}, y_{3}\right)= & l_{1} n_{1}^{2}+l_{2} n_{1} n_{3}+l_{3} n_{1} n_{2}+l_{4} n_{3}^{2} \\
& +l_{5} n_{2} n_{3}+l_{6} n_{1} n_{3}^{2}+l_{7} n_{1}^{2} n_{3}+l_{8} n_{1}^{3} \\
& +l_{9} n_{1} n_{2} n_{3}+l_{10} n_{1}^{2} n_{2}+l_{11} n_{3}^{3} \\
& +l_{12} n_{2} n_{3}^{2} ;
\end{aligned}
$$

$f^{2}\left(y_{1}, y_{2}, y_{3}\right)=l_{13} n_{1} n_{2}+l_{14} n_{2}^{2}+l_{15} n_{2} n_{3}+l_{16} n_{3}^{2}+$ $l_{17} n_{1} n_{3}+l_{18} n_{1}^{2}+l_{19} n_{2} n_{3}^{2}+l_{20} n_{1} n_{2} n_{3}+l_{21} n_{1}^{2} n_{2}+$ $l_{22} n_{2}^{3}+l_{23} n_{2}^{2} n_{3}+l_{24} n_{3}^{3}+l_{25} n_{1} n_{3}^{2}+l_{26} n_{1}^{2} n_{3}+l_{27} n_{1}^{3}$;

$f^{3}\left(y_{1}, y_{2}, y_{3}\right)=l_{28} n_{2}^{2}+l_{29} n_{2} n_{3}+l_{30} n_{3}^{2}+l_{31} n_{2}^{3}+$

$l_{32} n_{3}^{3}+l_{33} n_{2}^{2} n_{3}+l_{34} n_{2} n_{3}^{2}$;

where,

$$
\begin{gathered}
n_{1}=\left(a_{22} a_{33}-a_{23} a_{32}-v^{2}\right) y_{1}+v\left(a_{22}+a_{33}\right) y_{2} \\
+\left(\left(a_{22}+A_{1}\right)\left(a_{33}+A_{1}\right)-a_{23} a_{32}\right) y_{3} \\
n_{2}=-a_{21} a_{33} y_{1}-v a_{21} y_{2}-a_{21}\left(a_{33}+A_{1}\right) y_{3}, \\
n_{3}=a_{21} a_{32} y_{1}+a_{21} a_{32} y_{3}
\end{gathered}
$$

Equation (16) is the normal form of (10) from which the stability coefficient can be computed. In (10), on the right hand side the first term is linear and second one is nonlinear in $y^{\prime} s$. For evaluating the direction of bifurcating solution, we can evaluate the following quantities at $a_{2}=a_{2}^{*}$ and origin

$$
\begin{gathered}
g_{11}=\frac{1}{4}\left(C_{11}+i D_{11}\right) \\
g_{02}=\frac{1}{4}\left(C_{02}+i D_{02}\right) \\
g_{20}=\frac{1}{4}\left(C_{20}+i D_{20}\right) \\
G_{21}=\frac{1}{8}\left(C_{21}+i D_{21}\right) \\
C_{11}=\frac{\partial^{2} F^{1}}{\partial y_{1}^{2}}+\frac{\partial^{2} F^{1}}{\partial y_{2}^{2}} \\
D_{11}=\frac{\partial^{2} F^{2}}{\partial y_{1}^{2}}+\frac{\partial^{2} F^{2}}{\partial y_{2}^{2}} \\
C_{02}=\frac{\partial^{2} F^{1}}{\partial y_{1}^{2}}-\frac{\partial^{2} F^{1}}{\partial y_{2}^{2}}-2 \frac{\partial^{2} F^{2}}{\partial y_{1} \partial y_{2}}
\end{gathered}
$$

$$
\begin{gathered}
D_{02}=\frac{\partial^{2} F^{2}}{\partial y_{1}^{2}}-\frac{\partial^{2} F^{2}}{\partial y_{2}^{2}}+2 \frac{\partial^{2} F^{1}}{\partial y_{1} \partial y_{2}} \\
C_{20}=\frac{\partial^{2} F^{1}}{\partial y_{1}^{2}}-\frac{\partial^{2} F^{1}}{\partial y_{2}^{2}}+2 \frac{\partial^{2} F^{2}}{\partial y_{1} \partial y_{2}} \\
D_{20}=\frac{\partial^{2} F^{2}}{\partial y_{1}^{2}}-\frac{\partial^{2} F^{2}}{\partial y_{2}^{2}}-2 \frac{\partial^{2} F^{1}}{\partial y_{1} \partial y_{2}} \\
C_{21}=\frac{\partial^{3} F^{1}}{\partial y_{1}^{3}}+\frac{\partial^{3} F^{2}}{\partial y_{2}^{3}}+\frac{\partial^{3} F^{2}}{\partial y_{1}^{2} \partial y_{2}}+\frac{\partial^{3} F^{1}}{\partial y_{1} \partial y_{2}^{2}} \\
D_{21}=\frac{\partial^{3} F^{2}}{\partial y_{1}^{3}}+\frac{\partial^{3} F^{1}}{\partial y_{2}^{3}}+\frac{\partial^{3} F^{1}}{\partial y_{1}^{2} \partial y_{2}}+\frac{\partial^{3} F^{2}}{\partial y_{1} \partial y_{2}^{2}} \\
h_{11}=\frac{1}{4}\left(\frac{\partial^{2} F^{3}}{\partial y_{1}^{2}}+\frac{\partial^{2} F^{3}}{\partial y_{2}^{2}}\right) \\
h_{20}=\frac{1}{4}\left(\frac{\partial^{2} F^{3}}{\partial y_{1}^{2}}-\frac{\partial^{2} F^{3}}{\partial y_{2}^{2}}-2 i \frac{\partial^{2} F^{3}}{\partial y_{1} \partial y_{2}}\right) \\
w_{11}=\frac{h_{11}}{A_{1}}, w_{20}=\frac{h_{20}}{A_{1}+2 i \sqrt{A_{2}}} \\
G_{110}=\frac{1}{2}\left(C_{110}+i D_{110}\right) \\
G_{101}=\frac{1}{2}\left(C_{101}+i D_{101}\right) \\
C_{110}=\frac{\partial^{2} F^{1}}{\partial y_{1} \partial y_{3}}+\frac{\partial^{2} F^{2}}{\partial y_{2} \partial y_{3}} \\
D_{110}=\frac{\partial^{2} F^{2}}{\partial y_{1} \partial y_{3}}-\frac{\partial^{2} F^{1}}{\partial y_{2} \partial y_{3}} \\
\frac{D_{1} F_{1}}{\partial y_{3}}-\frac{\partial^{2} F^{2}}{\partial y_{2} \partial y_{3}} \\
\frac{\partial^{2} F^{2}}{\partial y_{3}}+\frac{\partial^{2} F^{1}}{\partial y_{2} \partial y_{3}} \\
\left.w_{11}+G_{101} w_{20}\right) \\
C_{11} g_{02} g_{20} g_{21}
\end{gathered}
$$

Thus, we can determine $g_{11}, g_{02}, g_{20}, g_{21}$ from (18),(19),(20), and (22), respectively. Thus, we can compute the following values:

$$
\begin{gathered}
C_{1}(0)=\frac{i}{2 v}\left[g_{20} g_{11}-\left|g_{11}\right|^{2}-\frac{1}{3}\left|g_{02}\right|^{2}\right]+\frac{g_{21}}{2} \\
\mu_{2}=\frac{-\operatorname{Re} C_{1}(0)}{\operatorname{Re} \lambda^{\prime}\left(a_{2}^{*}\right)}, \beta_{2}=2 \operatorname{Re} C_{1}(0) \\
\tau_{2}=-\frac{\left(\operatorname{Im} C_{1}(0)+\mu_{2} \operatorname{Im} \lambda^{\prime}\left(a_{2}^{*}\right)\right)}{v a_{2}^{*}}
\end{gathered}
$$

which determine the qualities of bifurcation periodic solution in the center manifold at the critical value $a_{2}^{*}$.

Theorem 4.2: The parameter $\mu_{2}$ determine the direction of the Hopf-bifurcation[15] if $\mu_{2}>0\left(\mu_{2}<0\right)$, then the Hopf-bifurcation is supercritical (subcritical) and the bifurcation periodic solutions exist for $a_{2}>a_{2}^{*}\left(a_{2}<a_{2}^{*}\right)$; $\beta_{2}$ determine the stability of bifurcating periodic solution, the bifurcation periodic solutions are orbitally asymptotically stable (unstable) if $\beta_{2}<0\left(\beta_{2}>0\right) ; \tau_{2}$ determine the period of the bifurcating periodic solution; the period increase (decrease) if $\tau_{2}>0\left(\tau_{2}<0\right)$.

\section{Boundedness of the System}

Lemma 5.1 All the solutions of system (4)-(5) with the positive initial condition are uniformly bounded within the 
region $\Omega_{1}$

where

$\Omega_{1}=\left\{(S, R): 0 \leq S+\frac{a_{1}}{b_{1}} R \leq \frac{2 r k}{\rho}, \rho=\min \left(r, d_{1}\right)\right\}$

is a region of attraction.

Proof. Proof is obvious.

Lemma 5.2 All the solutions of system (1)-(3) with the positive initial condition are uniformly bounded within the region $\Omega_{2}$

where

$\Omega_{2}=\left\{(S, R, C): 0 \leq S+\frac{a_{1}}{b_{1}} R+\frac{a_{1} a_{2}}{b_{1} b_{2}} C \leq \frac{2 r k}{\theta}, \theta=\right.$

minr, $d 1, d 2$

is a region of attraction.

Proof. We as sume that the right hand sides of the system (1)-(3) are smooth function of $(S(t), R(t), C(t))$ of $\left(t \in R_{+}\right)$. Let $S(t), R(t)$ and $C(t)$ be any solution with positive initial condition $(S(0), R(0), C(0))$.

From equation (1), we obtain

$$
\frac{d S}{d t} \leq r S\left(1-\frac{S}{k}\right)
$$

then by usual comparis on theorem[16]

$$
S \leq k
$$

We consider a time dependent function

$$
W(t)=S(t)+\frac{a_{1}}{b_{1}} R(t)+\frac{a_{1} a_{2}}{b_{1} b_{2}} C(t)
$$

The time derivative of $W(t)$ along the solution of the system (1)-(3) is

$$
\frac{d W}{d t}=r S\left(1-\frac{S}{k}\right)-\frac{a_{1} d_{1}}{b_{1}} R-\frac{a_{1} a_{2} d_{2}}{b_{1} b_{2}} C
$$

Since $S \leq K$, the above expression reduces to

$$
\frac{d W}{d t} \leq 2 r k-\frac{a_{1} d_{1}}{b_{1}} R-\frac{a_{1} a_{2} d_{2}}{b_{1} b_{2}} C-r S
$$

Taking $=\min \left(r, d_{1}, d_{2}\right)$, we get

$$
\frac{d W}{d t}+\theta W \leq 2 r k
$$

Applying comparis on theorem[17] we obtain

$$
0 \leq W(S, R, C) \leq \frac{2 r k}{\theta}+W(S(0), R(0), C(0)) e^{-\theta t}
$$

and for $t \rightarrow \infty$,

$$
0 \leq W(S, R, C) \leq \frac{2 r k}{\theta}
$$

Therefore all the solutions of system (1)-(3) in itiated at $(S(0), R(0), C(0))$ enter into the region

$$
\begin{aligned}
\Omega_{2}=\{(S, R, C): 0 & \leq S+\frac{a_{1}}{b_{1}} R+\frac{a_{1} a_{2}}{b_{1} b_{2}} C \leq \frac{2 r k}{\theta}, \\
\theta & \left.=\min \left(r, d_{1}, d_{2}\right)\right\}, \text { for any } \varepsilon>0 .
\end{aligned}
$$

Thus all the solutions of system (1)-(3) are uniformly bounded at $(S(0), R(0), C(0))$.

This completes the proof of the lemma.

\section{Global Stability Analysis of the Interior Equilibrium Point}

Theorem 6.1: The positive interior equilibrium point $E_{P}$ of the model 2 is globally asymptotically stable in absence of exotic predator species if $\frac{r}{k}>a_{1} R^{*}$ is satisfied.
Proof: Proof is obvious.

Theorem 6.2: If the following inequalities hold

$$
\begin{gathered}
H_{11}=\frac{r}{k}-\frac{a_{1} R^{*}\left(1+b C^{*}\right)}{1+\alpha R^{*}+\beta C^{*}}>0 \\
H_{22}=d_{1}-\frac{b_{1} h\left(1+b C^{*}\right)}{1+\alpha R^{*}+\beta C^{*}}+L_{1}>0 \\
H_{23}^{2}-H_{22} H_{33}<0
\end{gathered}
$$

where

$$
\begin{gathered}
L_{1}=\frac{H_{23}=L_{2}+L_{3}+L_{4}}{a_{2} C^{*}} \\
L_{3}=\frac{L_{2}=\frac{F_{1} b_{1} b S^{*} R^{*}(h+k)}{1+\alpha R^{*}+\beta C^{*}}}{\theta(h+k)\left(h+S^{*}\right)\left(1+\frac{2 b r k}{\theta}\right)\left(1+b C^{*}\right)} \\
L_{4}=\frac{2 r k F_{1} a_{2}\left(1+\alpha R^{*}\right)}{(h+k)\left(h+S^{*}\right)\left(1+\frac{2 b r k}{\theta}\right)\left(1+b C^{*}\right)} \\
H_{33}=\frac{F_{2} b_{2} \beta R^{*}}{(h+k)\left(h+S^{*}\right)\left(1+\frac{2 b r k}{\theta}\right)\left(1+b C^{*}\right)}
\end{gathered}
$$

$E_{P_{e}}$ is globally asymptotically stable with respect to solutions initiating in the interior of the positive orthant. Proof: Consider the following positive defin ite function

$$
\begin{gathered}
V(S, R, C)=\left(S-S^{*}-S^{*} \ln \frac{S}{S^{*}}\right)+\frac{F_{1}}{2}\left(R-R^{*}\right)^{2} \\
+F_{2}\left(C-C^{*}-C^{*} \ln \frac{C}{C^{*}}\right)
\end{gathered}
$$

where $F_{1}, F_{2}$ are positive constants to be chosen appropriately.

Differentiating $V$ with respect to $t$, we get

$$
\dot{V}=\frac{\left(S-S^{*}\right)}{S} \frac{d S}{d t}+F_{1}\left(R-R^{*}\right) \frac{d R}{d t}+F_{2} \frac{\left(C-C^{*}\right)}{C} \frac{d C}{d t}
$$

Substituting values of $\dot{S}, \dot{R}$ and $\dot{C}$ from the system of equations (1)-(3) in the above equation and after doing some algebraic manipulation, we get

$\dot{V}=-\left[C_{11}\left(S-S^{*}\right)^{2}+C_{22}\left(R-R^{*}\right)^{2}+C_{33}\left(C-C^{*}\right)^{2}+\right.$ $C 12 S-S * R-R *+C 13 S-S * C-C *+C 23 R-R * C-C *$

where

$$
\begin{gathered}
C_{11}=\frac{r}{k}-\frac{a_{1} R^{*}\left(1+b C^{*}\right)}{M}, C_{33}=\frac{F_{2} b_{2} \beta R^{*}}{N}, \\
C_{22}=F_{1}\left(d_{1}-\frac{b_{1}\left(1+b C^{*}\right)(h+S)}{M}+\frac{a_{2} C^{*}(1+\beta C)}{N}\right) \\
C_{12}=\frac{a_{1}\left(h+S^{*}\right)\left(1+b C^{*}\right)}{M}-\frac{F_{1} b_{1} h_{1} R\left(1+b C^{*}\right)}{M}, \\
C_{13}=-\frac{a_{1} b R^{*}(h+S)}{M}, \\
M=(1+\alpha R+\beta C)\left(1+\alpha R^{*}+\beta C^{*}\right), \\
N=(h+S)\left(h+S^{*}\right)(1+b C)\left(1+b C^{*}\right),
\end{gathered}
$$

Then a sufficient conditions[18] for $\dot{V}$ to be negative definite is that

$$
\begin{gathered}
C_{11}>0 \\
C_{22}>0 \\
C_{12}^{2}-C_{11} C_{22}<0 \\
C_{13}^{2}-C_{11} C_{33}<0 \\
C_{23}^{2}-C_{22} C_{33}<0
\end{gathered}
$$


hold.

Choosing $F_{1}$ and $F_{2}$ such a way

$$
\begin{gathered}
H_{12}^{2}<H_{11} H_{22} \\
H_{13}^{2}<H_{11} H_{33}
\end{gathered}
$$

where

$$
\begin{gathered}
H_{12}=\frac{a_{1}\left(h+S^{*}\right)\left(1+b C^{*}\right)}{1+\alpha R^{*}+\beta C^{*}}+\frac{2 r k F_{1} b_{1} h_{1}\left(1+b C^{*}\right)}{\theta\left(1+\alpha R^{*}+\beta C^{*}\right)} \\
H_{13}=\frac{a_{1} b R^{*}(h+k)}{1+\alpha R^{*}+\beta C^{*}}
\end{gathered}
$$

conditions (32) and (33) hold. However (32) implies (29) and (33) implies (30). Then above sufficient conditions reduce into the following inequalities.

$$
\begin{gathered}
C_{11}>0 \\
C_{22}>0 \\
C_{23}^{2}-C_{22} C_{33}<0
\end{gathered}
$$

However (24) implies (34), (25) implies (35) and (26) implies (36). Hence $\dot{V}$ is negative definite and so $\dot{V}$ is a Liapunov function with respect to $E_{P_{e}}$, proving the theorem.

\section{Simulation Analysis}

We have gained analytical understanding of possible dynamics of the native prey-predator, exotic predator model. We now perform some simulation work for the model 2 with set of parameters.

$r=0.5 ; k=16 ; a_{1}=0.4 ; b_{1}=0.36 ; d_{1}=0.15, h=9$.

For this choice of parameters we get a unique co-existing equilibrium point $E_{P}(6.4247,11.5357)$ of Model 2 along with $B_{1}>0, B_{2}>0$ and hence $E_{P}$ is locally asymptotically stable (see Figure 1(a), 1(b)). We now perform simulation work for the model 1 with set of parameters.

$$
r=0.5 ; k=16 ; \alpha=0.2 ; \beta=0.45 ; b=0.1 ; a_{1}=0.9 ;
$$

$a_{2}=0.138 ; b_{1}=0.2 ; b_{2}=0.051, d_{1}=0.00015, h=5.5$, $d_{2}=0.05$;

For this choice of parameters we get a unique co-existing equilibrium $E_{P_{e}}(12.9901,2.3193,2.0040)$ of Model 1 along with $A_{1}>0, A_{2}>0, A_{3}>0, A_{1} A_{2}-A_{3}>0$ and hence $E_{P_{e}}$ is locally asymptotically stable (see Figure 4(a), 4(b)). Now, we discuss the dynamical behaviour of the model 1 by varying the parameter $a_{2}$ taking the above set of parametric values. For this, we find out the positive roots of the equation $A_{1} A_{2}-A_{3}=0$ and we obtain three positive roots of this equation i.e. $a_{2}^{*}(1)=0.003955, a_{2}^{*}(2)=$ $0.01949, a_{2}^{*}(3)=0.1726$ (See Figure 7(a), 7(b), 7(c)). At $a_{2}^{*}$, one pair of eigen values of the characteristic equation (13) are of the form $\lambda_{1,2}= \pm i v$, where $v$ is a positive real number and hence the system (1)-(3) undergoes a Hopf-bifurcation at $a_{2}^{*}(1)=0.003955<a_{2}<a_{2}^{*}(2)=$ 0.01949 . For $a_{2}^{*}(1)=0.003955$, we get $C_{1}(0)=$ $-5.79886 \times 10^{-9}-1.56886 \times 10^{-7} i, u^{\prime}\left(a_{2}^{*}(1)\right)=$ 1.96078. From these values, it follows from (23) that $\mu_{2}>0$ and $\beta_{2}<0$ showing that the bifurcation takes place when $a_{2}$ crosses $a_{2}^{*}(1)$ to the right $\left(a_{2}^{*}(1)<a_{2}<\right.$ $\left.a_{2}^{*}(2)\right)$ and the corresponding periodic orbits are orbitally asymptotically stable (See Figure 5(a) and 5(b) for $a_{2}=$ $0.0041)$. For $a_{2}^{*}(2)=0.01949$, we get $C_{1}(0)=$ $2.96735 \times 10^{-8}+7.46404 \times 10^{-9} i, u^{\prime}\left(a_{2}^{*}(2)\right)=$

1.76203. From these values, it follows from (23) that $\mu_{2}<0$ and $\beta_{2}>0$ showing that the bifurcation takes when $a_{2}$ crosses $a_{2}^{*}(2)$ to the left $\left(a_{2}^{*}(1)<a_{2}<a_{2}^{*}(2)\right)$ and the corresponding periodic orbits are orbitally asymptotically unstable. The system (1)-(3) undergoes a Hopf-bifurcation at $a_{2}>a_{2}^{*}(3)$. For $a_{2}^{*}(3)=0.1726$ we get $C_{1}(0)=$ $-2.11192 \times 10^{-11}-8.89601 \times 10^{-11} i, \quad u^{\prime}\left(a_{2}^{*}(3)\right)=$ 1.96078. From these values, it follow from (23) that $\mu_{2}>0$ and $\beta_{2}<0$ showing that the bifurcation takes when $a_{2}$ crosses $a_{2}^{*}(3)$ to the right $a_{2}>a_{2}^{*}(3)$, and the corresponding periodic orbits are orbitally asymptotically stable (See Figure 6(a) and 6(b) $a_{2}=0.2$ ).

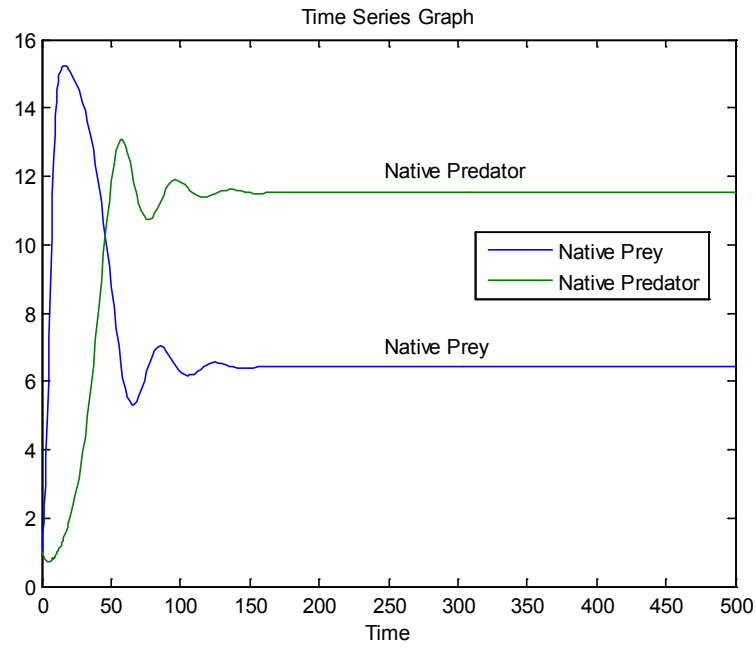

Figure 1(a). Stable population distribution for native prey and native predator species

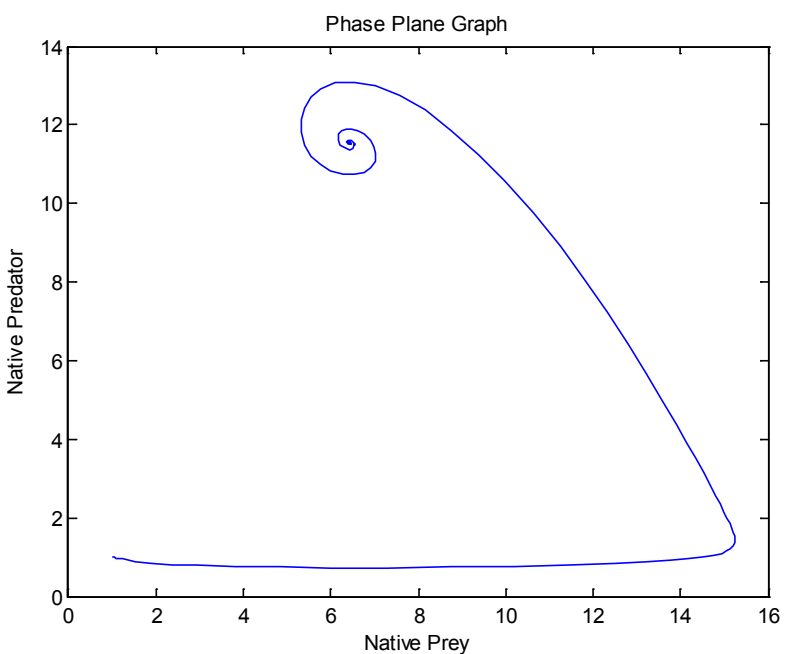

Figure 1(b). Phase space graph between native prey and native predator species 


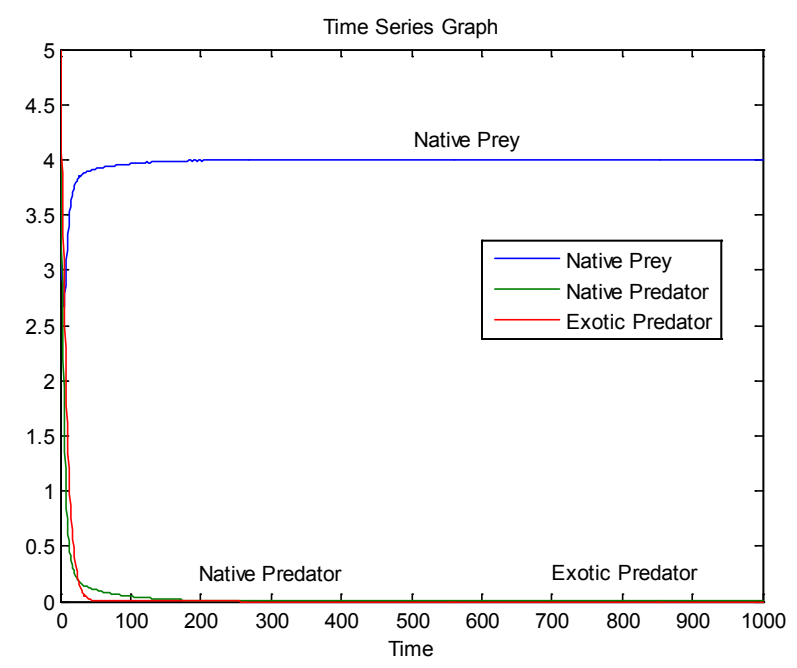

Figure 2. Time series graph for native prey, native predator and exotic predator species

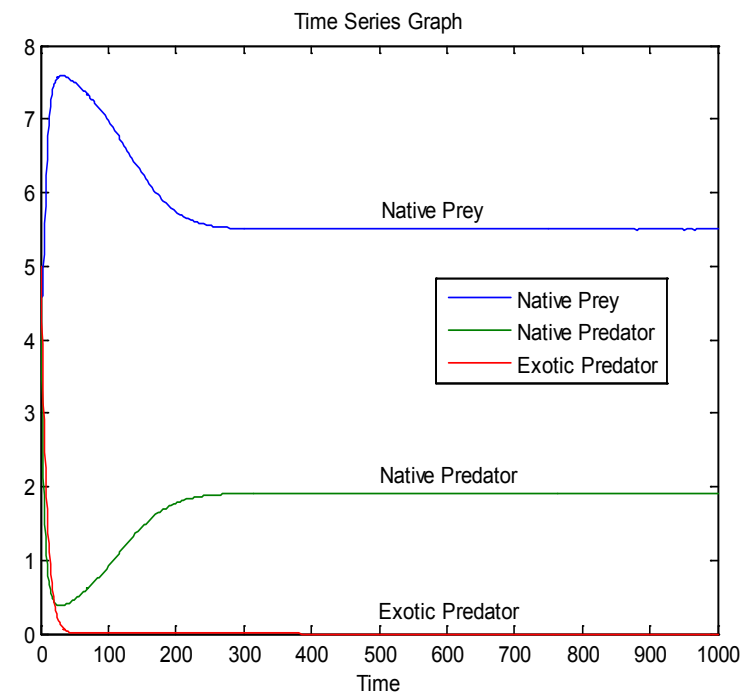

Figure 3. Time series graph for native prey, native predator and exotic predator species

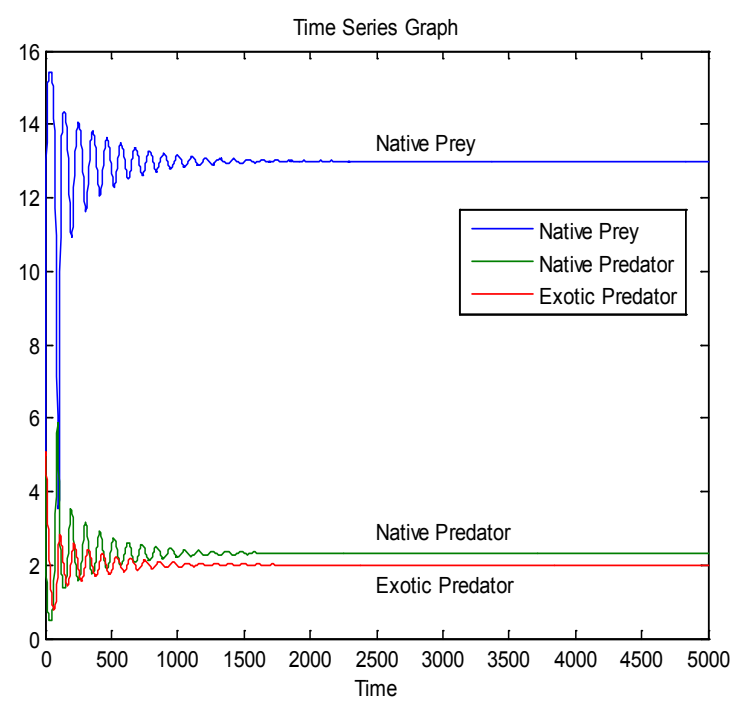

Figure 4(a). Stable population distribution for native prey, native predator and exotic predator species

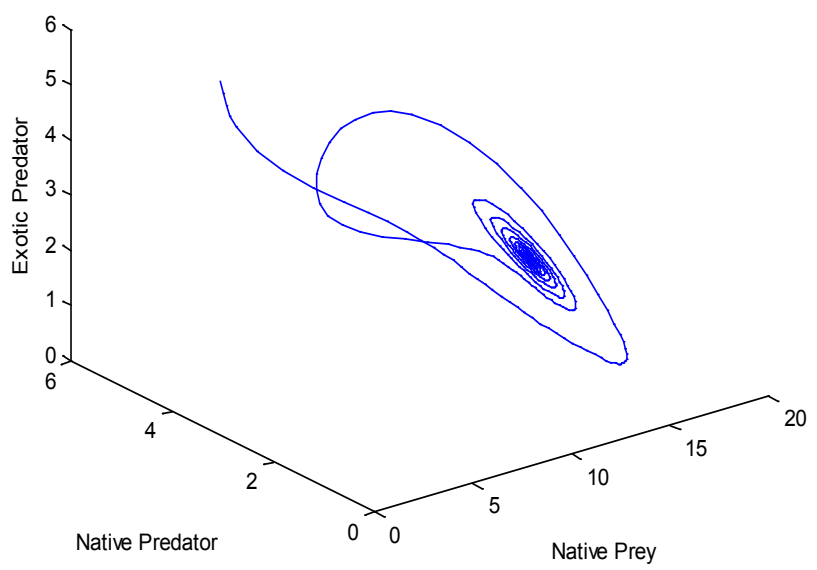

Figure 4(b). Phase space graph for native prey, nat ive predator and exotic predator species

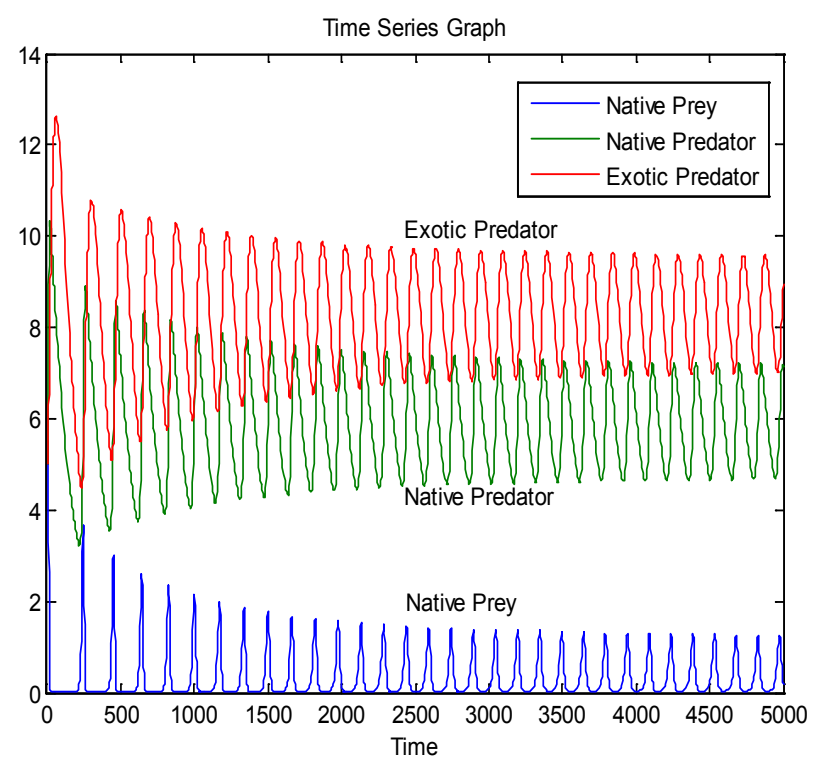

Figure 5(a). Time series graph for native prey, native predat or and exotic predator species at $a_{2}=0.0041$

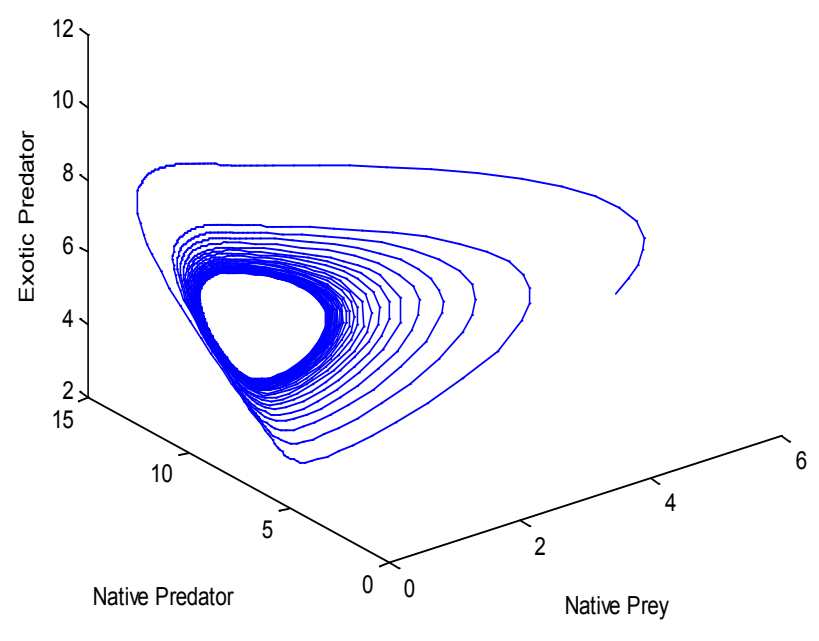

Figure 5(b). Phase space graph for native prey, nat ive predator and ex ot ic predator species at $a_{2}=0.0041$ 


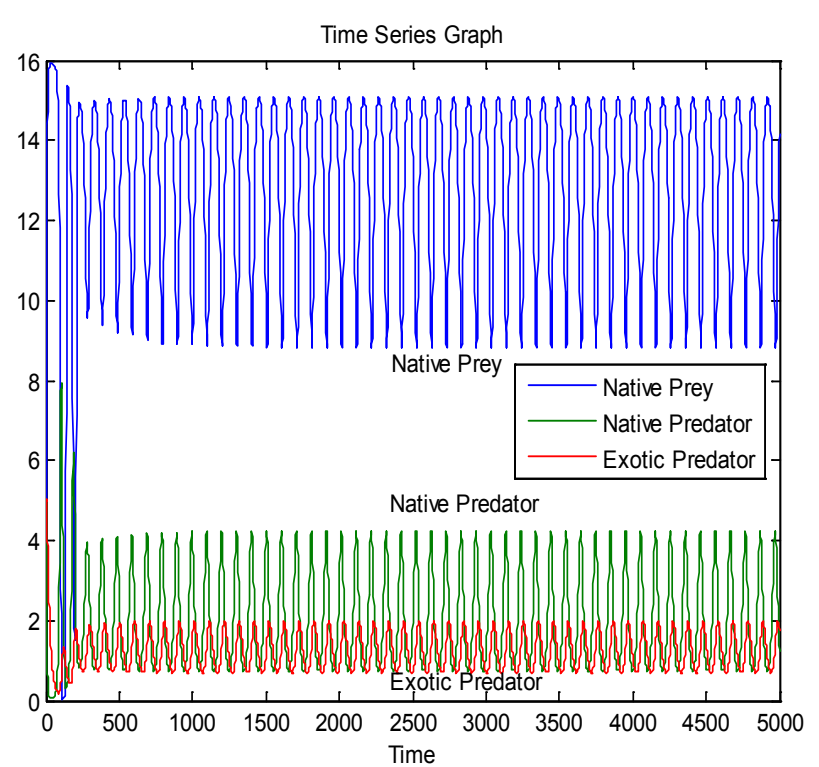

Figure 6(a). Time series graph for native prey, native predat or and exot ic predator species at $a_{2}=0.2$

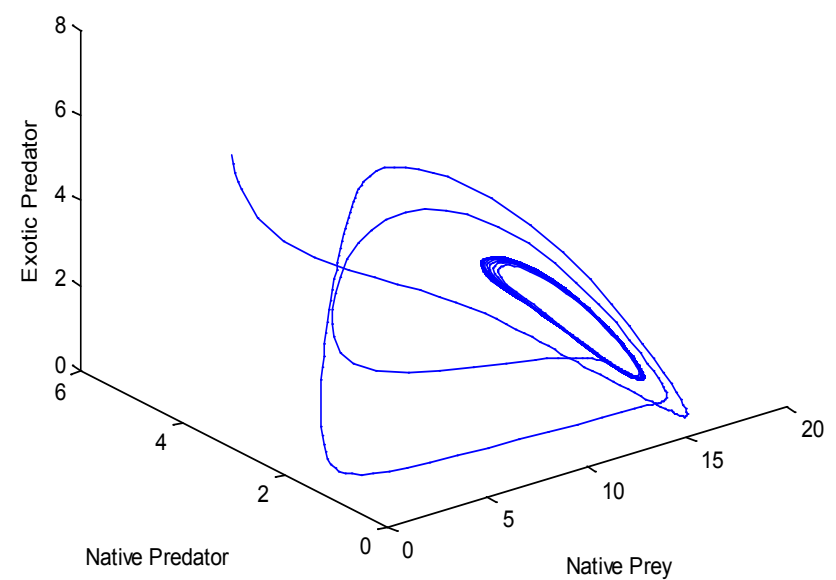

Figure 6(b). Phase space graph for nativeprey, native predator and exot ic predator species at $a_{2}=0.2$

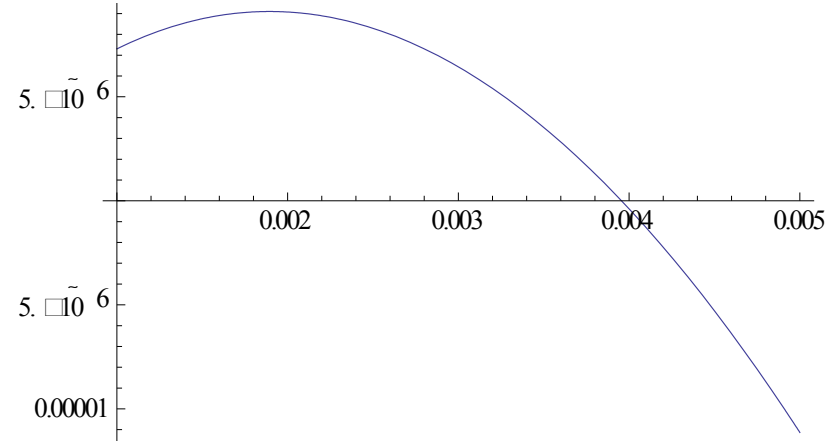

(a)

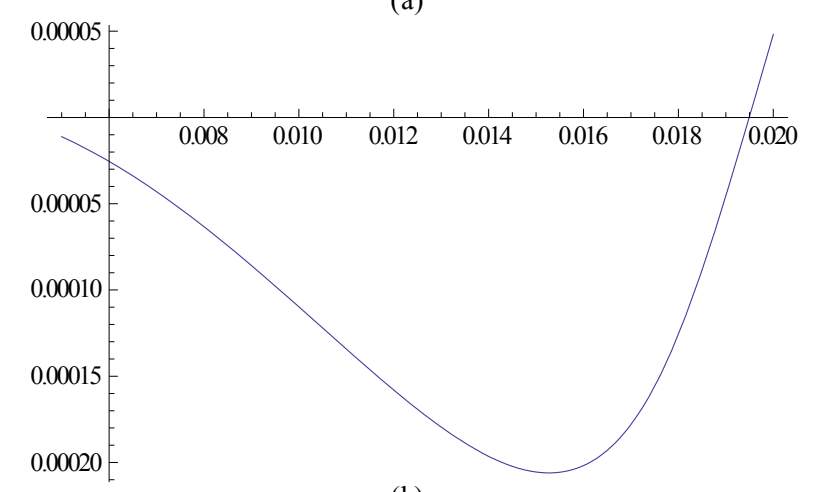

(b)

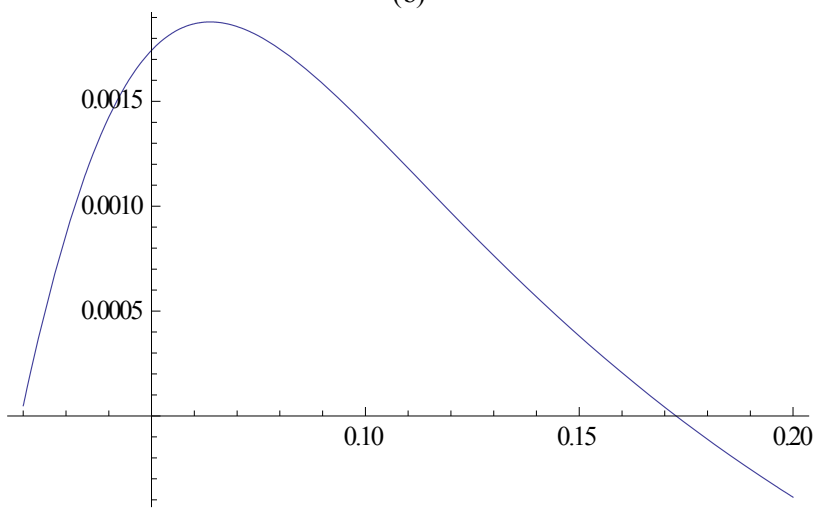

(c)

Figure 7. (a), (b), (c): Graph of $A_{1} A_{2}-A_{3}$ with respect to $a_{2}$

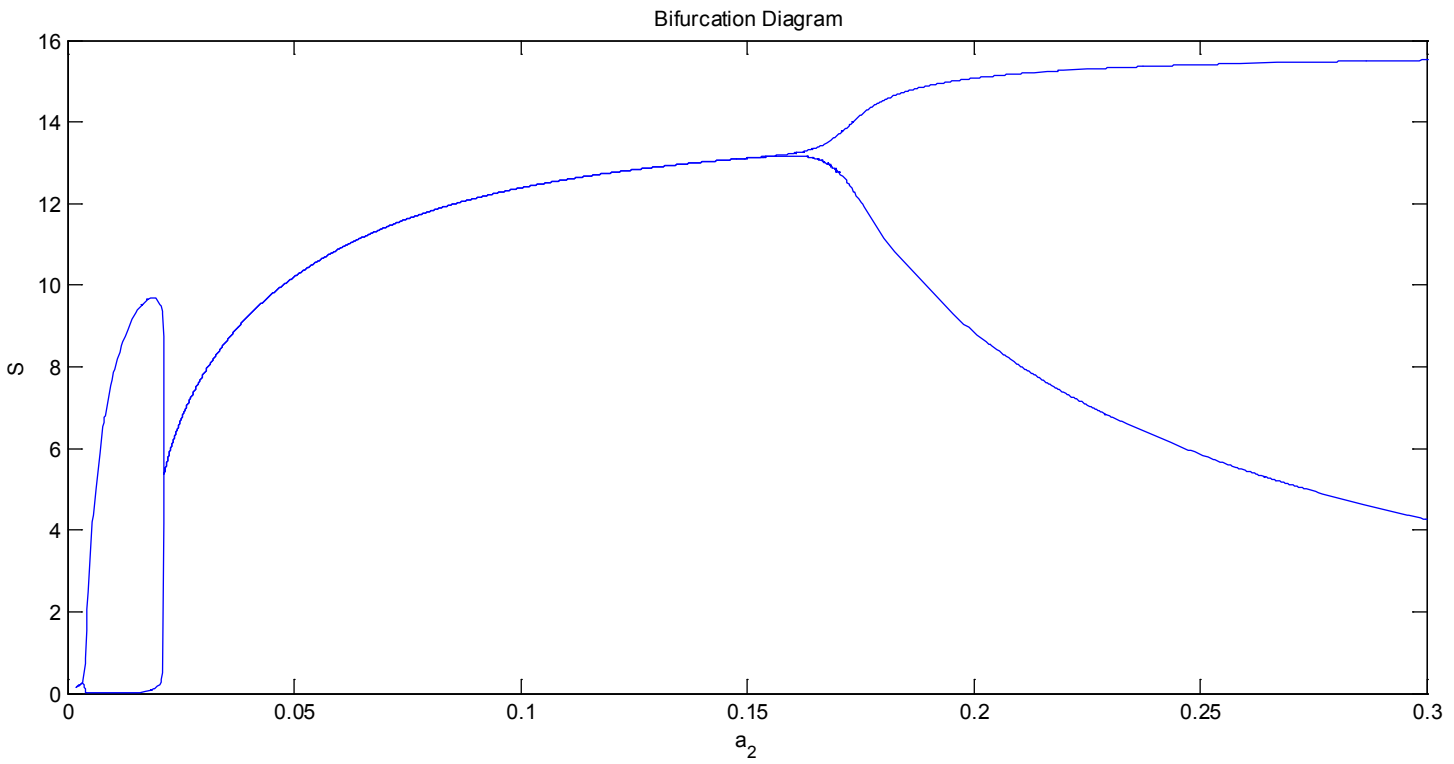

Figure 8(a). Bifurcation diagram of the native prey species with respect to predation rate $a_{2}$ 


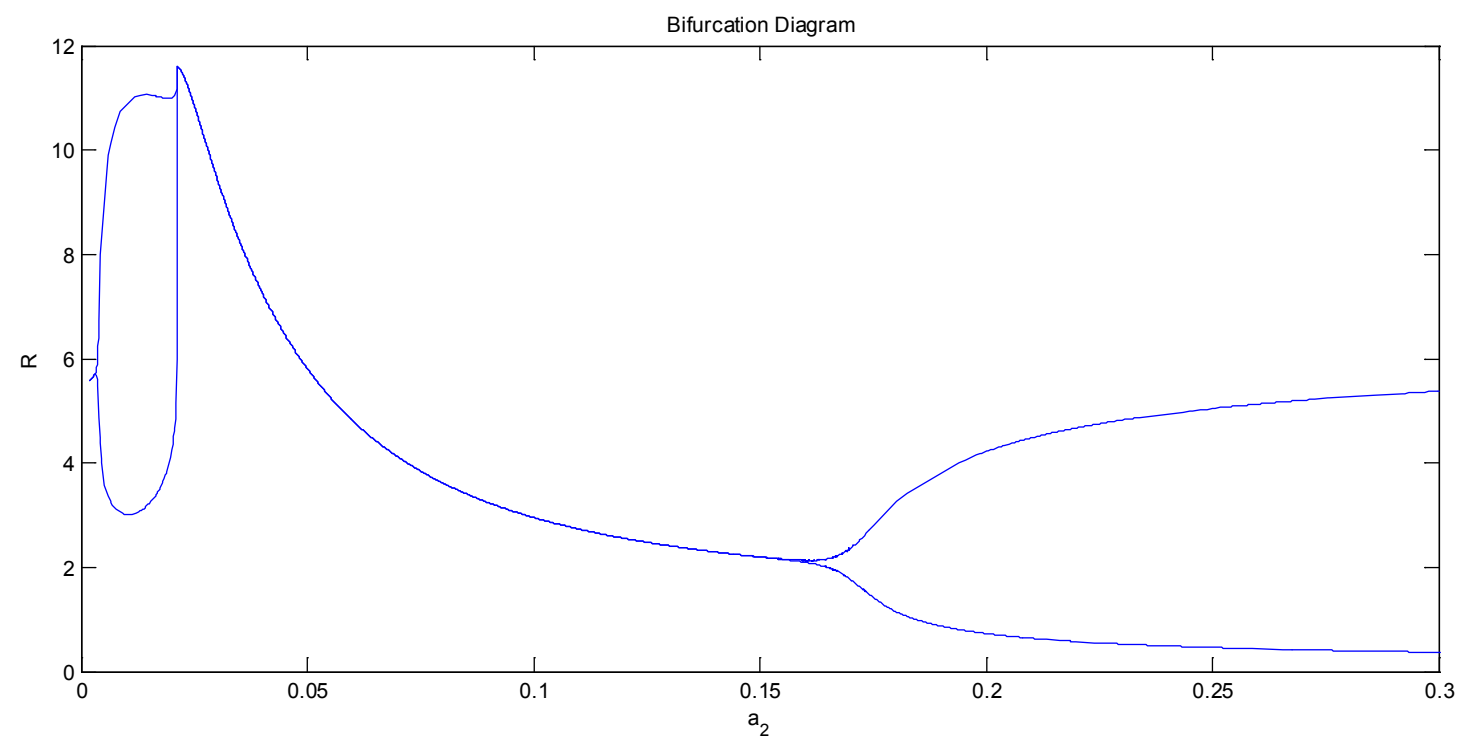

Figure 8(b). Bifurcation diagram of the native predat or species with respect to predation rate $a_{2}$

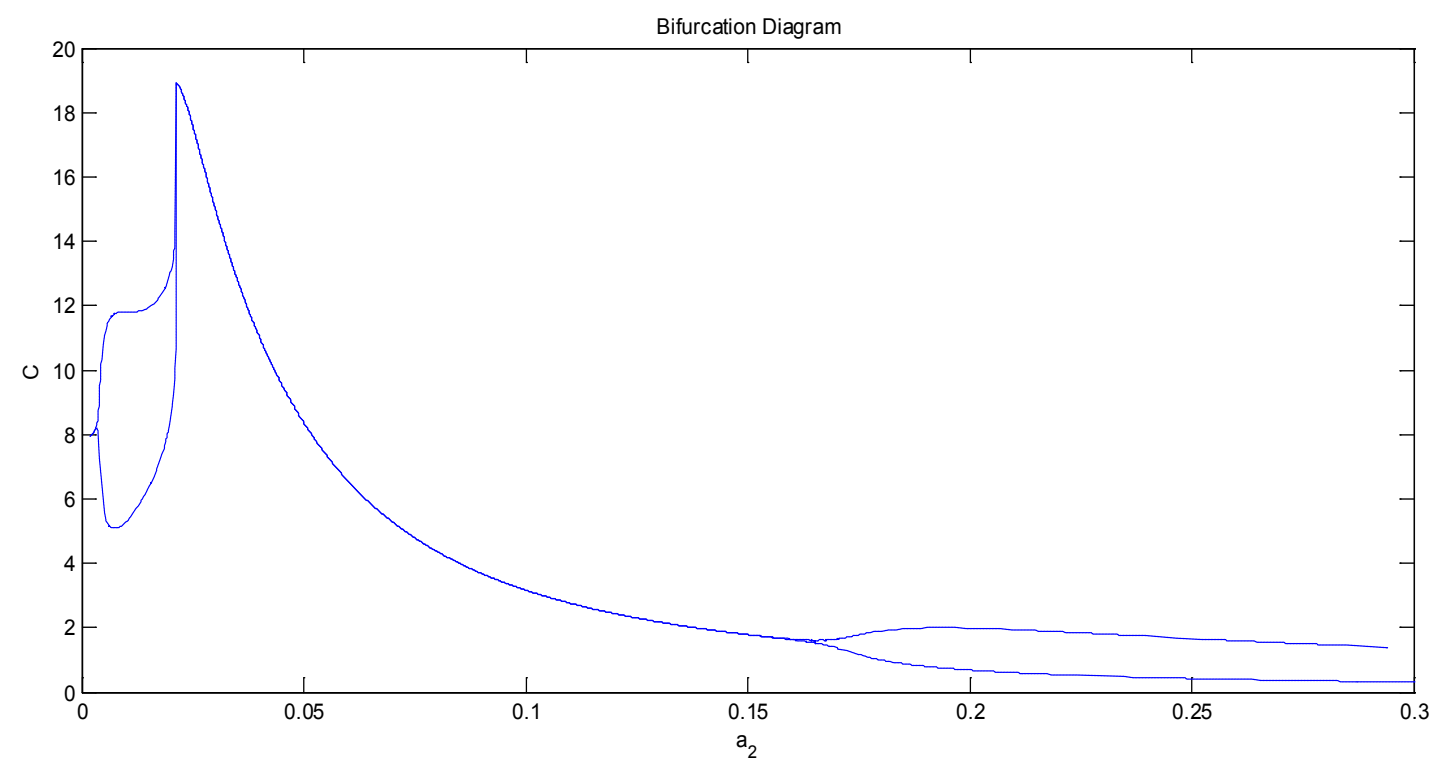

Figure 8(c). Bifurcation diagram of the exotic predator species with respect to predation rate $a_{2}$

\section{Conclusions}

In this paper, a mathematical model is proposed to study the effect of exotic predator population on a native prey-predator species system. The local stability analyses of all the feasible equilibria are carried out. We observed that if the axial equilibrium point $E_{A}$ of model 2 is stable then interior equilibrium point $E_{P}$ does not exist and if interior equilibrium point $E_{P}$ of model 2 exists then axial equilibrium point $E_{A}$ is unstable. From the stability of axial equilibrium po int $\mathrm{E}_{\mathrm{Ae}}$ [see figure 2], it may be concluded that native prey population will survive and native and exotic population may go to extinction. From the stability of boundary equilibrium point $\mathrm{E}_{\mathrm{Be}}$ [see figure 3], it is observed that the exotic predator population will not survive and consequently native prey-predator population will coexist. The global stability analysis of both the models 1 and 2 are carried out, and the possibility of Hopf- bifurcation of the interior equilibrium point is investigated. We observed that the positive interior equilibrium point $E_{P_{e}}$ is stable for $0<a_{2}<a_{2}^{*}(1)$ and $a_{2}^{*}(2)<a_{2}<a_{2}^{*}(3)$ (see figure 8(a), 8(b), 8(c)). The switching in stability behaviour based on predation rate of exotic predator; $a_{2}$ is also observed (see figure $8(\mathrm{a}), 8(\mathrm{~b}), 8(\mathrm{c}))$. We also determine the stability and direction of periodic bifurcation from the positive equilibrium at the critical point. Finally we conclude that invasion of exotic predator is harmful to native prey-predator system. We also clear that exotic predator is harmful to native predator and helpful to native prey. The predation rate of exotic predator creates complexphenomena in the system. The present work may be extended by considering the migration of the population. 


\section{REFERENCES}

[1] Alejandro Rojas-Palma, Eduardo González-Olivares, "Optimal harvesting in a predator-prey model with Allee effect and sigmoid functional response" , Applied Mathematical Modelling, 36, 1864-1874, 2012.

[2] Mainul Haque, "Existence of complex patterns in the Beddington-DeAngel is predator-prey model", Mathematical Biosciences, 239, 179-190, 2012.

[3] A. Braza Peter, "Predator-prey dynamics with square root functional responses", Nonlinear Analysis: Real World Applications 1,3,1837-1843, 2012.

[4] I. A. E. Atkinson, "Introduced animals and extinctions.. In:Western, D., Pearl, M.C. (Eds.), Conservation for the Twenty-first Century", Oxford University Press, New York, pp. 54-75, 1989.

[5] J. Diamond, "Overview of recent extinctions, In : Western, D., Pearl, M.C. (Eds.), conservation for the twenty-first century", Oxford university press, New York, 37-41, 1989.

[6] P. J. Moors, I. A. E. Atkinson, "Predation on seabirds by introduced animals, and factors affecting its severity. In : Croxall, J.P., Evans, P.G.H., Schreiber, R.W. (Eds.), Status and Conservation of theWorld's Seabirds", ICBP Technical Publication, No. 2, Cambridge, pp. 667-690, 1984.

[7] I. A. E. Atkinson, "Opportunities for ecological restoration", New Zealand Journal of Ecology, 11, 1-12, 1988.

[8] Jimin Zhang, Meng Fan, Yang Kuang, "Rabbits killing birds revisited", Mathematical Biosciences 203, 100-123, 2006.

[9] Meng Fan, Yang Kuang, Zhilan Feng, "Cats protecting birds revisited", Bulletin of mathematical biology, 67, 1081-1106, 2005.
[10] Zhenbu Zhang, "Qualitative Analysis for a Prey-Mesopredator-Superpredator Model", Applied Mathematical Sciences, Vol. 2, no. 42, 2063 - 2080, 2008.

[11] Raid Kamel Naji, Ranjit Kumar Upadhyay, Vikas Rai, "Dynamical consequences of predator interference in a tri-trophic model food chain", Nonlinear Analysis: Real World Applications, 11, 809-818, 2010.

[12] Xitao Wang, Min Zhao, "Chaos in a Hybrid Three-Species Food Chain with Beddington-Deangelis Functional Response", Procedia Environmental Sciences, 10, 128-134, 2011.

[13] Rimpi Pal, Debanjana Basu, M. Banerjee, "Modelling of phytoplankton allelopathy with Monod-Haldane-type functional response- A Mathematical Study", Biosy stem, 95, 243-253, 2009.

[14] W. M. Liu, "Criterion of Hopf-bifurcation without using eigenvalues”, J.Math. Anal. Appl., 182, 250-256, 1994.

[15] Swati khare, O. P. Misra, Chhatrapal Singh, Joydip Dhar, "Role of delay on planktonic ecosystem in the presence of a toxic producing phutoplankton", International journal of differential equation, 1-16, 2011.

[16] J. K. Hale, “Ordinary Differential Equation”, 2nd Ed, Kriegor, Basel,1980.

[17] G. Birkhoff, G. C. Rota, "Ordinary Differential Equation", Ginn. and Co, 1982.

[18] B. Dubey, R. K. Upadhyay, "Persistence and Extinction of One-Prey and Two-Predators system", Nonlinear analysis: Modelling and Control, 9, 307-329, 2004. 